\title{
The clc element of Pseudomonas sp. strain B13 and other mobile degradative elements employing phage-like integrases
}

Received: 4 September 2000 / Revised: 10 November 2000 / Accepted: 24 November 2000 / Published online: 25 January 2001

(C) Springer-Verlag 2001

\begin{abstract}
Genes for metabolic pathways in bacteria that degrade aromatic or aliphatic pollutants have mostly been confined to either plasmid DNAs or to the chromosome. For a few pathways, including classical pathways for chlorocatechol and biphenyl degradation, recent evidence has been obtained for location of the pathway genes on mobile DNA elements which employ phage-like integrases. This enables the DNA elements to integrate into specific sites on the chromosome and yet to excise and transfer to other host bacteria. This mini-review gives an overview of those elements and their relationship to an increasing number of phage-like elements associated with bacterial virulence.
\end{abstract}

Keywords clc Element - Pseudomonas sp. strain B13 . Chlorocatechol degradation - Biphenyl degradation .

Gene island · Phage-like integrase

\section{Introduction}

Plasmids have traditionally been seen as the major elements involved in conjugal gene transfer, at least in gramnegative bacteria. Among the many different characteristics which have been localized on and distributed by plasmid DNAs are the genes for so-called catabolic or degradative pathways. The study of degradative pathways is important both from environmental and evolutionary viewpoints. Microorganisms that degrade organic compounds contribute to the removal of toxic and polluting substances,

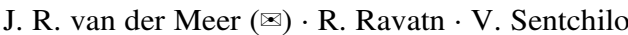

Swiss Federal Institute for Environmental Science and

Technology (EAWAG), Ueberlandstrasse 133, Postfach 611,

CH 8600, Dübendorf, Switzerland

e-mail: vdmeer@eawag.ch

Tel.: +41-1-8235438, Fax: +41-1-8235547

Present address:

R. Ravatn

The Cancer Institute of New Jersey

195 Little Albany Street, New Brunswick, NJ 08901, USA produced and disposed of by humans. Some of those degradation capabilities may in fact be very ancient. For example, it is reasonable to assume that microorganisms have faced aromatic and aliphatic compounds derived from fresh and fossilized plant material for ages. Therefore, pathways such as those for toluene, naphthalene, or alkane degradation, as we know them today, may have been present in bacteria for a long time. Degradation pathways for other compounds, especially those which are purely synthetic and have no direct natural counterparts (such as chlorinated alkanes and aromatic compounds) most likely reflect more recent evolutionary developments (Janssen et al. 1994; Schlömann 1994; van der Meer 1997; van der Meer et al. 1992). This evolution, however, mostly does not involve de novo generation of enzymes with specific capacities to deal with chlorinated compounds, but rather rearrangements of pre-existing gene fragments from different microorganisms. Since various chlorinated compounds are also naturally produced (De Jong et al. 1994), the enzymes which convert them must have been present in bacteria long before the environmental pollution by mankind and could thus be "recruited" to form other metabolic pathways. An example for such recruitment was found in bacteria using chlorobenzenes as sole carbon and energy source. These bacteria carried a specific new rearrangement between the genes for chlorocatechol degradation and a set of genes coding for a chlorobenzene dioxygenase and dihydrodiol dehydrogenase (Beil et al. 1999; van der Meer et al. 1992; van der Meer et al. 1998).

\section{The clc element of Pseudomonas sp. strain B13}

In an almost classical experiment, chlorobenzene-degrading organisms were also constructed in the laboratory by mimicking natural gene transfer (Oltmanns et al. 1988; Reineke et al. 1982). For this purpose, Pseudomonas sp. strain B13, a bacterium that metabolizes 3-chlorobenzoate (and carries the genes for chlorocatechol degradation), was mated with Pseudomonas putida F1, which degrades toluene. Bacteria using chlorobenzene as sole carbon and 
Fig. 1A, B Mechanism of sequence-specific integration of the $c l c$ element. A Under normal conditions in Pseudomonas sp. strain B13, the element is integrated in two gly-tRNA locations. The most distal 18 bp of the $g l y$-tRNA are repeated at the left end (shown as white and black triangles). The integrase gene (intB13) is located near the right end $(a t t R)$. Other phage-related proteins are encoded near the left end (attL). In the circular intermediate, the left and right ends are joined $(a t t P)$, generating a strong promoter for the integrase (arrow). B With a certain frequency, the $c l c$ element transfers to a new host and again integrates into the chromosomal target sequence ( $g l y$-tRNA gene). The 18-bp 3'-end of the gly tRNA gene is displaced to the left end, but the original $g l y$-tRNA sequence is restored by an identical 18-bp sequence of the clc element (open triangle)

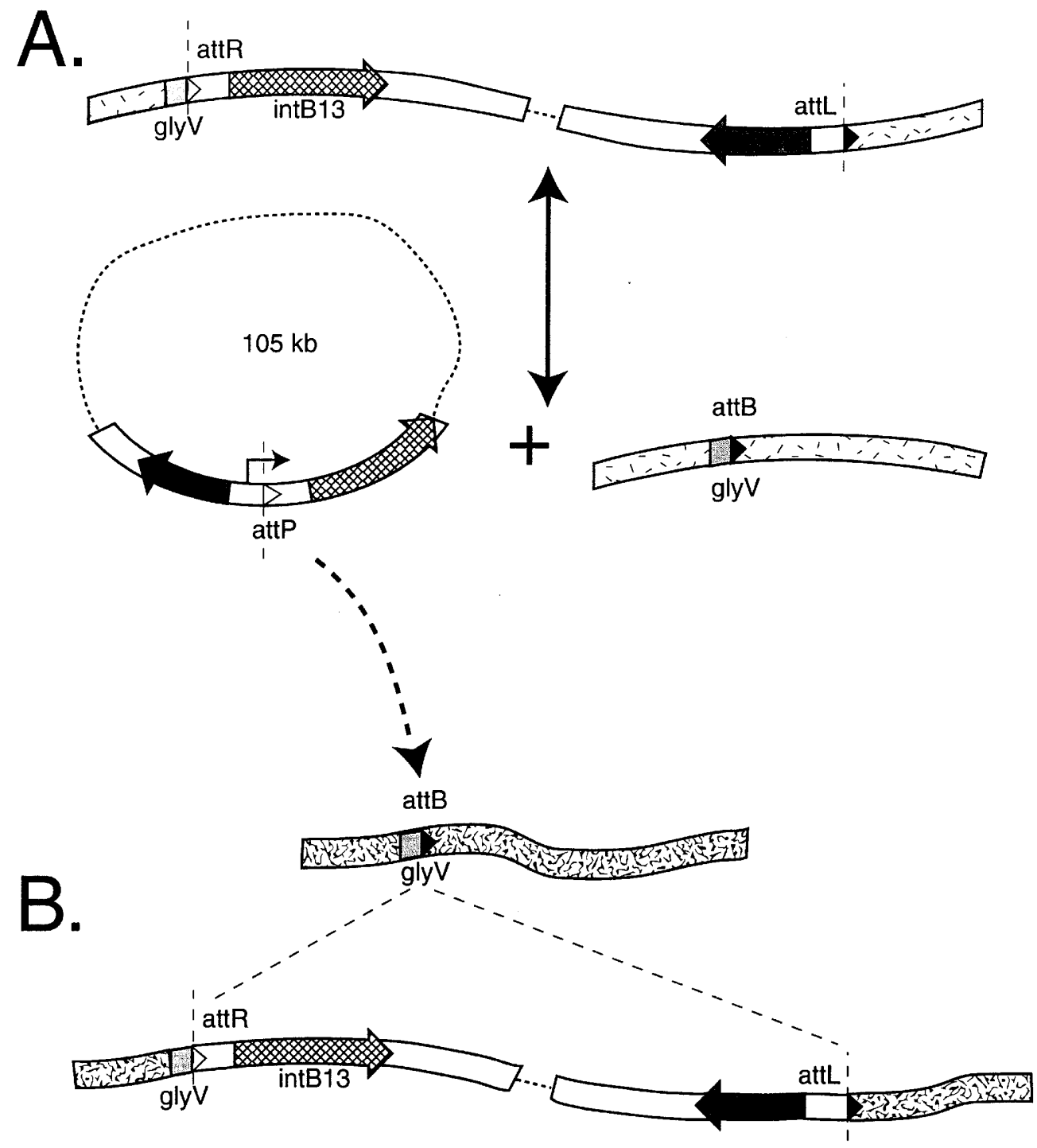

energy source were obtained which were of $P$. putida F1 origin, indicating that they had acquired a piece of DNA from strain B13 (Oltmanns et al. 1988). The same mating method was used to transfer the genes for chlorocatechol degradation from strain B13 to various other recipients (Reineke 1998). Until recently, it had always been suspected that the genes were transferred from strain B13 by means of a conjugative plasmid, pB13 (Chatterjee and Chakrabarty 1983). This plasmid had been described once; however, independent isolation remained unsuccessful (Weisshaar et al. 1987).

More recently, upon repetition of this mating experiment, we found that the transferred element was not a classical plasmid. In fact, in $P$. putida $\mathrm{F} 1$, the transferred DNA behaved rather strangely and formed large, unstable duplications that were integrated into the chromosome at two locations (Ravatn et al. 1998a). The transferred DNA was at that point named the $c l c$ element (for its first-described characteristic: chlorocatechol degradation). Subsequent analysis of strain B13 itself showed that the element was also present in the chromosome, in two separate locations (Ravatn et al. 1998a). This indicated that the element acted as an integrative plasmid or perhaps as a conjugative transposon. The size of the transferred element was determined from large chromosomal fragment mapping and from cosmid libraries as $105 \mathrm{~kb}$ (Ravatn et al. 1998a). Restriction-site mapping indicated an almost perfect match to the published physical map of the described plasmid pB13, which suggested that the $c l c$ element could be present in two forms, as free plasmid and integrated. Since the element was present in two locations on the chromosome, we were able to compare both integration sites and to determine the ends of the element. This indicated that the right end was contained near or in a gene for glycine-tRNA $(g l y V)$, whereas the left end was formed by an 18-bp sequence repeated from the right end (Fig. 1). About $250 \mathrm{bp}$ downstream from the right end, we found a large ORF coding for a phage-type integrase (Ravatn et al. 1998b). This suggested that the clc element was not just an integrated plasmid, but employed an integrase to achieve site-specific integration. Activity of the integrase could be shown in Escherichia coli by using a two-plasmid system: 
one plasmid with the integrase gene plus attP (like in the circular form of the element) and another plasmid with the reconstructed $a t t B$ site (containing the glycine tRNA gene) (Ravatn et al. 1998b). Integrase activity from the attR configuration (like in the integrated situation) was not detected.

\section{Bacteriophage-related genetic elements}

From the analyses described above, it became clear that the $c l c$ element is a bacteriophage-related element. Such elements are a loosely defined group of DNA elements, including some bacteriophages, prophages, insertional plasmids, conjugative transposons and "gene islands" [most of which are known as pathogenicity islands (PAI)]. The distinctions and definitions given in the literature sometimes become rather diffuse and artificial. In fact, it seems as if a spectrum of different mobilization and transfer mechanisms is being utilized. All these elements are related by their means of site-specific recombination, involving integrase and (in some cases) excisionase enzymes. Apart from this, however, the remaining DNA of the element may encode totally different functions, varying from phage structural proteins to plant symbiotic factors or virulence determinants. For example, most genomes contain integrated prophages, although it is not always clear if they can be induced. Satellite phages, like P4 (Pierson and Kahn 1987) or the Staphylococcus aureus pathogenicity island SaPI (Ohlsen et al. 1999), require another phage (P2 for P4) for head assembly and transduction. Other bacteriophages (e.g., P1) have a plasmid origin of replication (Park et al. 1998); however, their mode of transfer is by transduction. Insertional plasmids, as found in Actinomycetes (Smokvina et al. 1991), seem to replicate autonomously in most hosts, but can also integrate into the chromosome. The IncJ element R391 from Proteus rettgeri behaves as an insertional plasmid. In E. coli, it is normally integrated at one unique site in the chromosome. However, the element has functional plasmid replicative and incompatibility systems, and plasmid forms can be isolated from recA strains in which the chromosomal site is occupied by a related element (Pembroke and Murphy 2000). Insertional plasmids use conjugation rather than transduction as means of transfer. Conjugative transposons (e.g., CTn916) mostly do not replicate independently, but form a circular intermediate form, from which conjugal transfer is initiated (Scott and Churchward 1995). PAIs mostly occur as unstable regions of the chromosome and carry virulence genes (Hacker and Kaper 1999). Some of them still have active integration functions and may even behave as satellite phages [such as the 40-kb VPI pathogenicity island of Vibrio cholerae (Karaolis et al. 1999)]. Other PAIs contain an integrase and a replication origin, like the 12-kb vap region of the gram-negative anaerobic pathogen Dichelobacter nodosus (Cheetham et al. 1995). Some PAIs, like the high pathogenicity island (HPI) of Yersinia pseudotuberculosis, still carry a phage-type integrase, but do not show evidence for functions involved in self-transmissability or replication (Buchrieser et al. 1998).
And in some PAIs, even the integrase function may have become lost (Hacker and Kaper 1999).

On the basis of similarities among integrase enzymes, five large groups have been recognized (Nunes-Düby et al. 1998). These consist of: (1) bacteriophage-type integrases, (2) resolvases, (3) transposases, (4) excisionase/integrases, and (5) invertases. All these proteins essentially maintain the active-site residues (the $\mathrm{HXXRX}_{28-35} \mathrm{Y}$ signature), although the overall amino acid sequence similarities may be insignificantly low (Nunes-Düby et al. 1998). The use of the term "integrase", therefore, causes considerable confusion, because it does not directly infer its specific mode of action. For example, resolvases will promote site-specific recombination between two copies of a specifically recognized direct repeat sequence on the same DNA. On the other hand, bacteriophage-type integrases promote cutting-out (excision) and subsequent integration into a new target DNA (not necessarily on the same DNA molecule). The integrase from the $c l c$ element belongs to the bacteriophage-type group (Ravatn et al. 1998b) and is related to what is called the phage P4 subfamily of integrases. These integrases are not only site-specific but also sequence-specific, and use tRNA structural genes as integration sites (Fig. 2). Interestingly, various other elements employ P4-type integrases (Fig.2), although there is no direct conservation in the targeted tRNA genes. P4-type integrases have been found in prophages themselves; in PAIs [such as the vap element of D. nodosus (Cheetham et al. 1995), or the HPI of Y. pseudotuberculosis (Buchrieser et al. 1998)], and in a "symbiosis island" in Mesorhizobium loti (Sullivan and Ronson 1998). The symbiosis island encompasses approximately $500 \mathrm{~kb}$ and contains the genes necessary for nodule formation, nitrogen fixation, and synthesis of three vitamins.

The percentages of amino acid identity and similarity of the IntB13 integrase are moderately low (37-55\%) with respect to other P4-type integrases (Fig. 2) and are limited to an N-terminal region of approximately 420 amino acids. In addition, the integrase of the $c l c$ element is about 250 amino acids longer than most other P4-type integrases. This was puzzling, since only two other sequences were available which showed significant similarity to this C-terminal extension (Fig. 2). It might be that these are actually part of a larger ORF, which has not yet been determined. Recently, however, an integrase sequence almost fully identical to IntB13 was discovered in the genome sequence of the plant pathogen Xylella fastidiosa (Silvestri et al. 2000). This part, however, was not recognized as a prophage or as a potential gene island. The X. fastidiosa region also showed a gly-tRNA gene nearby the integrase gene, similar to the $c l c$ element, which might form the right end of the $X$. fastidiosa gene island (Fig. 3). Screening of the downstream $X$. fastidiosa sequence revealed that at three locations possible repetitions of the 18 -bp recognition sequence of the gly-tRNA gene are found: at 66.6, 71.6 , and $365 \mathrm{~kb}$ distance downstream of the gly-tRNA gene (GenBank entry AE004839). The sequence at a distance of $71.6 \mathrm{~kb}$ from the $g l y$-tRNA gene had again strong similarity with the left end of the clc element. This sug- 
Pseudomonas B13

X. fastidiosa

$P$. aeruginosa

$X$. fastidiosa

S. flexneri

E. coli CP4-like

Y. pseudotuberculosis

D. nodosus

R. eutropha Tn4371
AJ004950

$\bigwedge$ tRNA-gly

intB13

AE003995

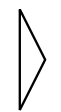

tRNA-gly

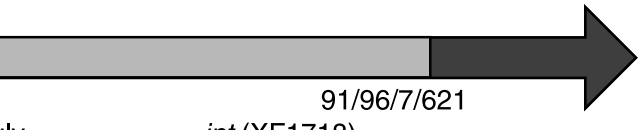

int (XF1718)

XF1719

D84146

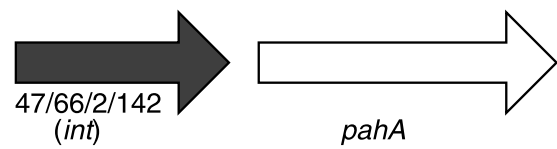

AJ251217

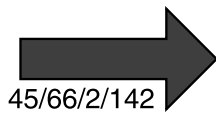

(int)

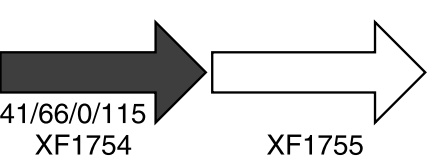

AF141323
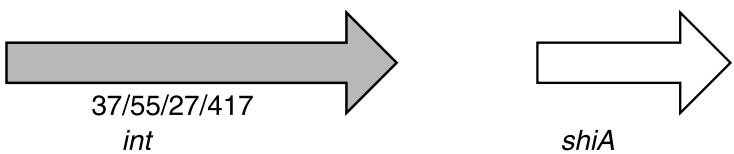

shiA

AF071034

tRNA-sec
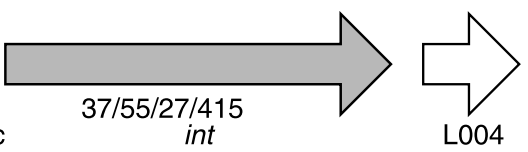

AJ009592

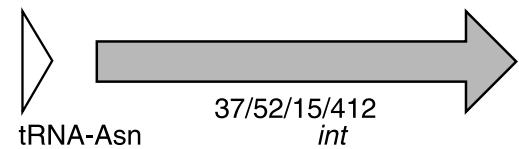

AF049243
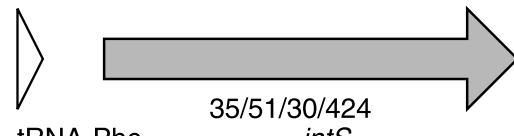

tRNA-Phe

ints

L31763
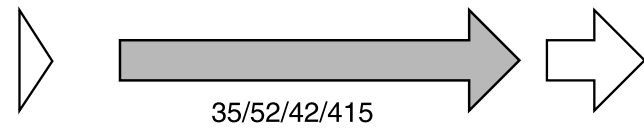

tRNA-Ser

int $A$

$\operatorname{vapG}$

Y10831

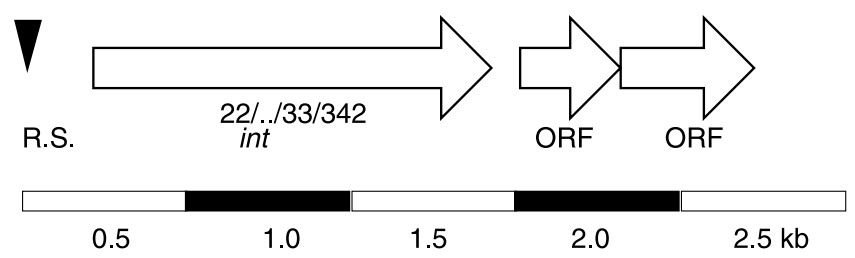

Fig. 2 Comparison of the gene organizations of integrases related to IntB13 of Pseudomonas sp. strain B13 and of integrases in other degradative pathways. Arrows indicate sizes and direction of the major ORFs. Gene names or gene assignments are indicated directly below the ORFs. At the far left, the bacteria in which the elements, phages, or conjugative transposons were originally found and the GenBank accession numbers are listed. Note that both intB13 and int(XF1718) are considerably longer than other P4type integrases and, in fact, are longer than most other known integrases (Nunes-Düby et al. 1998). Shading in gray corresponds to those parts that are similar to P4-type integrases. Shading in black corresponds to a few genes (or parts of genes) that exclusively are similar to the C-terminal ends of intB13 and int(XF1718). Numbers below the integrase ORFs result from a comparison of these ORFs to the Int B13 integrase. They indicate \% identity, \% similarity, number of gaps, and length of the overlap for the deduced amino acid sequences, respectively. The vertical black triangle in the $\mathrm{Tn} 4371$ gene organization depicts the location of the recombination site (R.S.) 
gests that this might be the left end of an integrated gene island in this region of the $X$. fastidiosa genome. Most of the ORFs on this gene island encode hypothetical proteins without clear similarities to others (Silvestri et al. 2000). However, some ORFs indicate relationships to conjugative transfer capabilities. It will be interesting to know more about the sequence of the $c l c$ element and to compare it with that of the $X$. fastidiosa element. Immediately downstream of the integrase gene, however, there is no obvious sequence identity between these elements, and the X. fastidiosa sequence does not carry the clc genes for chlorocatechol degradation. Interestingly, a similar (putative) protein comprising only the C-domain of both IntB13 and $X$. fastidiosa integrase is also present elsewhere on the $X$. fastidiosa genome (Fig. 2), which suggests that this ORF might have become recombined with an ancestral P4-type integrase.

\section{Integration mechanism}

There is too little information to draw conclusions on all details of integration, excision, and transfer of the clc element. Nonetheless, based on experiments and similarities to other systems, we can propose the following mechanisms. In all hosts analyzed so far, the $c l c$ element is present in the integrated form in one, two, or more copies
(Fig. 3) (Ravatn et al. 1998a, b). With a certain low frequency, the element excises, which results in a circular intermediate. Whether this molecule is capable of replicating remains to be determined. The fact that the clc element previously had been characterized as a plasmid pB13 (Chatterjee and Chakrabarty 1983) might indicate that in some hosts replication may occur. Also, the observation of multiple tandem copies integrated in the chromosome of P. putida F1 might point to replication (Ravatn et al. 1998a). Analysis of the attP region on the circular form indicated that both ends are connected here. No indication was found for a heterologous hybrid as formed in the circular intermediate of conjugative transposons (Scott and Churchward 1995). The attP sequence carries an identical 18-bp sequence as the $a t t B$ site of the target, which seems to be the actual site of recombination. If the $c l c$ element works in a manner similar to conjugative transposons, we should assume that the element excises precisely from its original location and that the cuts introduced in this area are ligated. There may well be an equilibrium state between the excised and the integrated form. With a certain

Fig. 3 Comparison of the integration sites for the $c l c$ element in different recipient bacteria and for the (putative) Xylella fastidiosa element. For reasons unknown, the element integrates only into gly-tRNA genes that carry a downstream sequence of strong diad symmetry. The black arrow points to the actual site of insertion
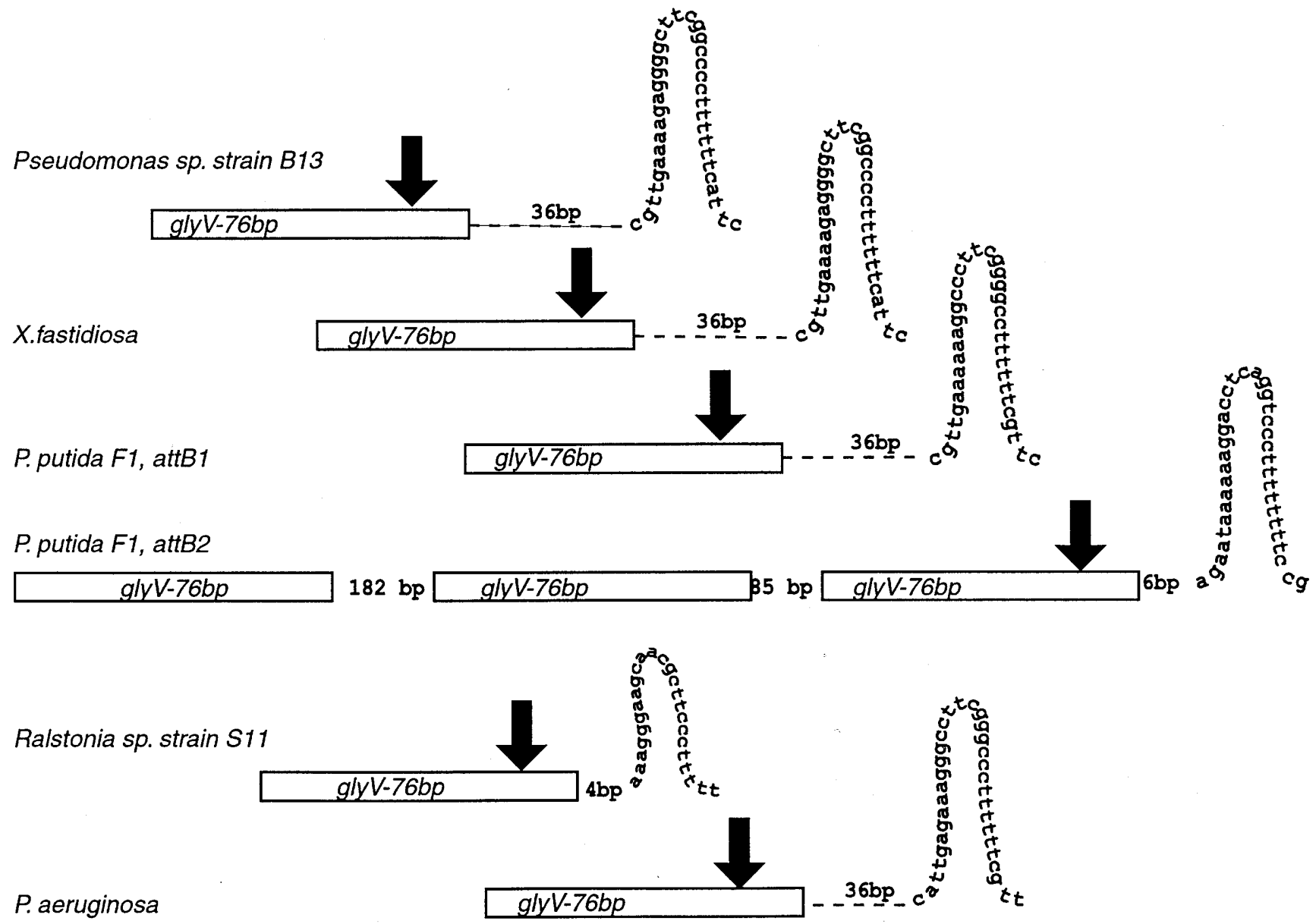
frequency, however, the element transfers to another recipient (Ravatn et al. 1998c). The transfer mechanism is basically unknown; in fact, theoretically it is still possible that the $c l c$ element is transduced rather than conjugated. In the new host, the element integrates very effectively, since connecting both ends in the circular form creates a strong promoter for the integrase (Ravatn et al. 1998b). A prerequisite for the target site is a gly-tRNA gene and, probably, some sort of hairpin structure immediately downstream of the target. This can be concluded from the different hosts in which the clc element has integrated. For example, Pseudomonas aeruginosa has three copies for $g l y$-tRNA; however, only the one with the downstream hairpin is targeted. Pseudomonas putida has four copies for $g l y$-tRNA, but only two of them (carrying the hairpin structure) are used as integration sites. X. fastidiosa (Silvestri et al. 2000) has two copies of the gly-tRNA gene, but only in the one with the hairpin structure is the IntB13like integrase present.

Very little is known about possible regulation of excision or transfer. Since transfer of some plasmids [e.g., Tiplasmid (Winans 1992)] or conjugative transposons [e.g., NBU1 of Bacteroides sp. (Stevens et al. 1992)] is regulated by signal molecules in the environment and/or by the status or density of the cells themselves, something similar might be expected for the $c l c$ element. The single observation made in our laboratory so far is that the amount of the circular form detectable in growing cultures of Pseudomonas sp. strain B13 significantly increases only when the cells are grown on 3-chlorobenzoate.

\section{Is the clc element a curiosity or the tip of an iceberg?}

As long as the clc element has only one close homologue (IntXF1718), it remains a curiosity. However, there is every reason to assume that DNA elements which employ phage-like integrase functions play a role in catabolic gene transfer and pathway evolution. The first reason is that, despite the fact that there has been no description of other homologues of the $c l c$ element encoding catabolic pathways, there are a few comparable systems (Fig. 2). Secondly, the literature shows some evidence for plasmids with unexpected integration capacities [e.g., Ka and Tiedje (1994); Pembroke and Murphy (2000)].

We know of at least one other bacterium carrying an element very strongly related to the $c l c$ element (van der Meer, unpublished data). This bacterium, Ralstonia sp. strain JS705, was isolated from contaminated groundwater in the USA. It grows on chlorobenzene and 3-chlorobenzoate and carries the $c l c$ genes for chlorocatechol degradation (van der Meer et al. 1998). Another example for a mobilizable chromosomal element with catabolic genes comes from a recent investigation of the biphenyl-degrading organism P. putida KF715 (Nishi et al. 2000). This 90-kb conjugative element was first discovered as an unstable chromosomal region. Deletions were found to arise spontaneously that involved loss of either or both the $b p h$ and sal genes (for biphenyl and salicylate degradation, respec- tively) or large-scale chromosomal rearrangements. Mating resulted in the transfer of both $b p h$ and sal genes from the original strain to $P$. putida AC30, and from this strain to $P$. putida KT2440. In both recipient organisms, the element could be targeted to the chromosome. Unfortunately, no information is yet available on the characterization of the genes for integration, or on mobilization, the target site, or the mode of action of this element. It might be that the bph-sal element is related to the other biphenyl mobile element Tn4371 (see below) or to the partial integrase sequences of P. aeruginosa and Pseudomonas sp. strain B4 (Fig. 2), which are also located near naphthalene and biphenyl degradation genes.

The presence of a phage-type integrase was demonstrated for the transposon structure Tn4371 in Ralstonia eutropha (Merlin et al. 1999; Springael et al. 1993). The transposon is $55 \mathrm{~kb}$ in size and carries a $13-\mathrm{kb}$ region with the $b p h$ genes for biphenyl degradation. The right end contains a 40-kb second transposon, tn- $b p h$. At the left end, an integrase gene is found (Fig. 2). This integrase, however, has very little overall sequence similarity to IntB13, but rather belongs to the phage P22 subfamily. Tn4371 inserts relatively site-specifically into a TTTTTCAT sequence, which is present on RP4 in two sites, and in five sites in the chromosome of $R$. eutropha. The transposon does not form cointegrates, indicating that it has a cut-and-paste mode of transposition rather than a replicative mode. A circular intermediate of $\mathrm{Tn} 4371$ was detected by PCR. Precise excision was observed from the kanamycin resistance gene within RP4, which was restored exactly. Although the right end of Tn4371 contains two ORFs with similarity to Ti-membrane transfer proteins, no independent conjugation of $\mathrm{Tn} 4371$ has yet been detected (Merlin et al. 1999). All the characteristics of Tn4371 suggest that it is a conjugative transposon and, therefore, different from the clc element, which targets a tRNA gene.

Only in three other bacteria with degradative pathways have there been descriptions of possible integrase functions, to the best of our knowledge. Two of these (i.e., in Pseudomonas pavonacea and Mycobacterium sp. G1) were of gene regions for a dehalogenase involved in haloalkane degradation (Poelarends et al. 2000). The other concerned the catabolic plasmid pNL1 of Sphingomonas aromaticivorans (Romine et al. 1999). However, all of these integrases belong to the "resolvase" class, rather than the bacteriophage-class.

\section{Concluding remarks}

Pseudomonas sp. strain B13 carries a transferable element, which is unique for bacteria that degrade aromatic or aliphatic pollutants. This element, which employs a phage P4-like integrase, is related to a family of elements that are widespread among bacteria (Fig. 2). Therefore, it might be expected that more of these elements will be discovered in association with degradation pathways. However, it cannot be a priori excluded that the higher incidence of phage-like elements in pathogenic bacteria not only reflects 
a bias in the research effort, but somehow results from selection. Perhaps phage-like elements are just not as effective in the evolution and selection of degradation pathways as the plasmid themselves.

\section{References}

Beil S, Timmis KN, Pieper DH (1999) Genetic and biochemical analyses of the tec operon suggest a route for evolution of chlorobenzene degradation genes. J Bacteriol 181:341-346

Buchrieser C, Brosch R, Bach S, Guiyoule A, Carniel E (1998) The high-pathogenicity island of Yersinia pseudotuberculosis can be inserted into any of the three chromosomal asn tRNA genes. Mol Microbiol 30:965-978

Chatterjee DK, Chakrabarty AM (1983) Genetic homology between independently isolated chlorobenzoate-degradative plasmids. J Bacteriol 153:532-534

Cheetham BF, Tattersall DB, Bloomfield GA, Rood JI, Katz ME (1995) Identification of a gene encoding a bacteriophage-related integrase in a vap region of the Dichelobacter nodosus genome. Gene 162:53-58

Hacker J, Kaper JB (1999) The concept of pathogenicity islands. In: Kaper JB, Hacker J (eds) Pathogenicitiy islands and other mobile virulence elements. American Society for Microbiology, Washington, DC, pp 1-12

De Jong E, Field JA, Spinnler H-E, Wijnberg JBPA, de Bont JAM (1994) Significant biogenesis of chlorinated aromatics by fungi in natural environments. Appl Environ Microbiol 60:264-270

Janssen DB, Pries F, van der Ploeg JR (1994) Genetics and biochemistry of dehalogenating enzymes. Annu Rev Microbiol 48:163-91

Ka JO, Tiedje JM (1994) Integration and excision of a 2,4dichlorophenoxyacetic acid-degradative plasmid in Alcaligenes paradoxus and evidence of its natural intergeneric transfer. J Bacteriol 176:5284-5289

Karaolis DK, Somara S, Maneval DR, Jr., Johnson JA, Kaper JB (1999) A bacteriophage encoding a pathogenicity island, a typeIV pilus and a phage receptor in cholera bacteria. Nature 399: 375-379

Merlin C, Springael D, Toussaint A (1999) Tn4371: A modular structure encoding a phage-like integrase, a Pseudomonas-like catabolic pathway, and RP4/Ti-like transfer functions. Plasmid 41:40-54

Nishi A, Tominaga K, Furukawa K (2000) A 90-kilobase conjugative chromosomal element coding for biphenyl and salicylate catabolism in Pseudomonas putida KF715. J Bacteriol 182: 1949-55

Nunes-Düby SE, Kwon HJ, Tirumalai RS, Ellenberger T, Landy A (1998) Similarities and differences among 105 members of the Int family of site-specific recombinases. Nucleic Acids Res 26:391-406

Ohlsen K, Ziebuhr W, Reichardt W, Witte W, G\&circ;tz F, Hacker J (1999) Mobile elements, phages, and genomic islands of staphylococci and streptococci. In: Kaper JB, Hacker J (eds) Pathogenicitiy islands and other mobile virulence elements. American Society for Microbiology, Washington, DC, pp 265-287

Oltmanns RH, Rast HG, Reineke W (1988) Degradation of 1,4dichlorobenzene by constructed and enriched strains. Appl. Microbiol. Biotechnol. 28:609-616

Park K, Mukhopadhyay S, Chattoraj DK (1998) Requirements for and regulation of origin opening of plasmid P1. J Biol Chem 273:24906-24911

Pembroke JT, Murphy DB (2000) Isolation and analysis of a circular form of the IncJ conjugative transposon-like elements, R391 and R997:implications for IncJ incompatibility. FEMS Microbiol Lett 187:133-138

Pierson LS, Kahn ML (1987) Integration of satellite bacteriophage P4 in Escherichia coli. DNA sequences of the phage and host regions involved in site-specific recombination. J Mol Biol 196: 487-496
Poelarends GJ, Kulakov LA, Larkin MJ, van Hylckama Vlieg JE, Janssen DB (2000) Roles of horizontal gene transfer and gene integration in evolution of 1,3-dichloropropene- and 1,2-dibromoethane-degradative pathways. J Bacteriol 182:2191-2199

Ravatn R, Studer S, Springael D, Zehnder AJ, van der Meer JR (1998a) Chromosomal integration, tandem amplification, and deamplification in Pseudomonas putida F1 of a 105-kilobase genetic element containing the chlorocatechol degradative genes from Pseudomonas sp. strain B13. J Bacteriol 180:4360-4369

Ravatn R, Studer S, Zehnder AJB, van der Meer JR (1998b) Int$\mathrm{B} 13$, an unusual site-specific recombinase of the bacteriophage P4 integrase family, is responsible for chromosomal insertion of the 105-kilobase clc element of Pseudomonas sp. strain B13. J Bacteriol 180:5505-5514.

Ravatn R, Zehnder AJB, van der Meer JR (1998c) Low-frequency horizontal transfer of an element containing the chlorocatechol degradation genes from Pseudomonas sp. strain B13 to Pseudomonas putida $\mathrm{F} 1$ and to indigenous bacteria in laboratory-scale activated-sludge microcosms. Appl Environ Microbiol 64:2126-2132

Reineke W (1998) Development of hybrid strains for the mineralization of chloroaromatics by patchwork assembly. Annu Rev Microbiol 52:287-331

Reineke W, Wessels SW, Rubio MA, Latorre J, Schwien U, Schmidt E, Schlömann M, Knackmuss HJ (1982) Degradation of monochlorinated aromatics following transfer of genes encoding chlorocatechol catabolism. FEMS Microbiol Lett 14:291-294

Romine MF, Stillwell LC, Wong KK, Thurston SJ, Sisk EC, Sensen C, Gaasterland T, Fredrickson JK, Saffer JD (1999) Complete sequence of a 184-kilobase catabolic plasmid from Sphingomonas aromaticivorans F199. J Bacteriol 181:1585-1602

Schlömann M (1994) Evolution of chlorocatechol catabolic pathways: conclusions to be drawn from comparisons of lactone hydrolases. Biodegradation 5:301-321

Scott JR, Churchward GG (1995) Conjugative transposition. Annu Rev Microbiol 49:367-397

Silvestri ML, Siqueira WJ, de Souza AA, de Souza AP, Terenzi MF, Truffi D, Tsai SM, Tsuhako MH, Vallada H, Van Sluys MA, Verjovski-Almeida S, Vettore AL, Zago MA, Zatz M, Meidanis J, Setubai JC (2000) The genome sequence of the plant pathogen Xylella fastidiosa. The Xylella fastidiosa consortium of the organization for nucleotide sequencing and analysis, Sao Paulo, Brazil. Nature 406:151-157

Smokvina T, Boccard F, Pernodet JL, Friedmann A, Guerineau M (1991) Functional analysis of the Streptomyces ambofaciens element pSAM2. Plasmid 25:40-52

Springael D, Kreps S, Mergeay M (1993) Identification of a catabolic transposon, Tn4371, carrying biphenyl and 4-chlorobiphenyl degradation genes in Alcaligenes eutrophus A5. J. Bacteriol. 175:1674-1681

Stevens AM, Sanders JM, Shoemaker NB, Salyers AA (1992) Genes involved in production of plasmid-like forms by a Bacteroides conjugal chromosomal element share amino acid homology with two-component regulatory systems. J Bacteriol 174:2935-2942

Sullivan JT, Ronson CW (1998) Evolution of rhizobia by acquisition of a $500-\mathrm{kb}$ symbiosis island that integrates into a phetRNA gene. Proc Natl Acad Sci USA 95:5145-5149

Van der Meer JR (1997) Evolution of novel metabolic pathways for the degradation of aromatic compounds. Anton von Leeuwenhoek Int J Microbiol 71:159-178

Van der Meer JR, de Vos WM, Harayama S, Zehnder AJB (1992) Molecular mechanisms of genetic adaptation to xenobiotic compounds. Microbiol Rev 56:677-694

Van der Meer JR, Werlen C, Nishino S, Spain JC (1998) Evolution of a pathway for chlorobenzene metabolism leads to natural atttenuation in a contaminated groundwater. Appl Environ Microbiol 64:4185-4193

Weisshaar M-P, Franklin FCH, Reineke W (1987) Molecular cloning and expression of the 3-chlorobenzoate-degrading genes from Pseudomonas sp. strain B13. J Bacteriol 169:394-402

Winans SC (1992) Two-way chemical signaling in Agrobacterium-plant interactions. Microbiol Rev 56:12-31 